\title{
What is the true definition of a "Do-Not- Resuscitate" order? A Japanese perspective
}

Eiji Hiraoka'

Yosuke Homma

Yasuhiro Norisue ${ }^{3}$

Takaki Naito'

Yuko Kataoka'

Osamu Hamada'

Yo Den'

Osamu Takahashi ${ }^{4}$

Shigeki Fujitani ${ }^{3}$

'Department of Internal Medicine, 2Department of Emergency Medicine, ${ }^{3}$ Department of Critical Care Medicine, Tokyo Bay Urayasu Ichikawa Medical Center, Chiba, Japan; ${ }^{4}$ Department of Internal Medicine, St Luke's International Hospital,

Tokyo, Japan
Correspondence: Eiji Hiraoka Department of Internal Medicine, Tokyo Bay Urayasu Ichikawa Medical Center, 3-4-32 Todaijima Urayasu, Chiba 279000I, Japan

$\mathrm{Tel}+8|4735| 3|0|$

Fax +8I 473526237

Email eijih@jadecom.jp
This article was published in the following Dove Press journal:

International Journal of General Medicine

29 June 2016

Number of times this article has been viewed

Background: Japan has no official guidelines for do-not-resuscitate (DNR) orders. Therefore, we investigated the effect of DNR orders on physician decision making in relation to performing noncardiopulmonary resuscitation (CPR) and CPR procedures.

Methods: A case-scenario-based questionnaire that included a case of advanced cancer, a case of advanced dementia, and a case of nonadvanced heart failure was administered to physicians. The questions determined whether physicians would perform different non-CPR procedures and CPR procedures in the presence or absence of DNR orders. The number of non-CPR procedures each physician would perform and the number of physicians who would perform each non-CPR and CPR procedure in the absence and presence of DNR ocrders were compared. Physicians from three Japanese municipal acute care hospitals participated.

Results: We analyzed 111 of 161 (69\%) questionnaires. Physicians would perform significantly fewer non-CPR procedures in the presence of DNR orders than in the absence of DNR orders for all three case scenarios (median [interquartile range] percentages: Case 1: 72\% [45\%-90\%] vs $100 \%$ [90\%-100\%]; Case 2: 55\% [36\%-72\%] vs 91\% [63\%-100\%]; Case 3: $78 \%$ [55\%-88\%] vs $100 \%$ [88\%-100\%]). Fewer physicians would perform non-CPR and CPR procedures in the presence of DNR orders than in the absence of DNR orders. However, considerable numbers of physicians would perform electric shock treatment for ventricular fibrillation in the presence of DNR orders (Case 1: 26\%; Case 2: 16\%; Case 3: 20\%).

Conclusion: DNR orders affect physician decision making about performing non-CPR procedures. Although some physicians would perform CPR for ventricular fibrillation in the presence of DNR orders, others would not. Therefore, a consensus definition for DNR orders should be developed in Japan, otherwise DNR orders may cause harm.

Keywords: code status, do-not-resuscitate order (DNR)

\section{Background}

Cardiopulmonary resuscitation (CPR) was introduced in the USA around $1960 .{ }^{1}$ In 1976, the first hospital policies regarding do-not-resuscitate (DNR) orders were established. ${ }^{1}$ Since then, CPR has become the default standard of care in the USA, unless a DNR order has been written with a patient's consent. ${ }^{1,2}$

DNR orders are intended to allow patients to forgo CPR in the event of cardiac arrest $^{3}$ and cannot be applied to any situation other than cardiac arrest. The American Medical Association's Council on Ethical and Judicial Affairs has even published guidelines stating that "DNR orders only preclude resuscitative efforts and should not influence other therapeutic interventions that may be appropriate". ${ }^{3,4}$ Nonetheless, 
DNR orders have been applied to procedures that include evidence-based life-prolonging treatments other than CPR for cardiac arrest. Indeed, patients in the USA who had DNR orders and were admitted for acute heart failure were less likely to undergo an assessment of their left ventricular function, to receive treatment with angiotensin-converting enzyme inhibitors or angiotensin II receptor blockers, or to undergo counseling about lifestyle modifications. ${ }^{5}$ In addition, a survey of hospital residents and physicians in the USA that used case scenarios depicting patients at the final stages of cancer and HIV infection showed that, in the presence of DNR orders, physicians were less likely to order tests and treatments that were not related to $\mathrm{CPR} .^{3}$ An additional report showed that a physician hesitated to order a blood transfusion for a patient with gastrointestinal bleeding who had a DNR order. ${ }^{6}$

In Japan, guidelines regarding DNR orders have yet to be developed, and in most hospitals, including our own, there is no obligation to order a code status when a patient is admitted to the hospital. However, the Japanese society is aging rapidly, and, indeed, the Cabinet Office of the Government of Japan reported that the percentage of the population that was aged $\geq 65$ years was $24.1 \%$ in 2012 , but it is forecast to exceed $30 \%$ in $2025 .{ }^{7}$ Furthermore, investigations have revealed that $>90 \%$ of the people aged $\geq 65$ years would request natural deaths without life-prolonging treatment in life-threatening situations if there was no prospect of recovery. ${ }^{7}$ Therefore, a code status ordering system needs to be established in Japan to prevent unwanted CPR.

Some physicians have started ordering DNR instructions for some terminally ill patients, but there are no reports that state exactly what percentage of Japanese physicians have ordered these instructions and how they affect physician decision making with respect to performing non-CPR procedures. Although DNR orders should be applied to CPR procedures only, they are sometimes regarded as comfort measures in routine medical practice, and there is a lingering fear that the presence of DNR orders might lead to the omission of life-saving non-CPR procedures, especially invasive procedures. ${ }^{3,4}$ Furthermore, although ventricular fibrillation (VF), pulseless ventricular tachycardia, asystole, and pulseless electrical activity (PEA) indicate cardiac arrest and DNR orders prevent CPR, ${ }^{8}$ physicians' understandings of the definitions of cardiac arrest and DNR must be ascertained in Japan. Therefore, we performed a cross-sectional survey using case scenarios that included diagnostic tests and treatment options to investigate the effects of DNR orders on physician decision making regarding the performance of
non-CPR and CPR procedures as well as physicians' understandings of cardiac arrest and DNR orders.

\section{Methods}

\section{Study design}

We performed a cross-sectional survey of physicians using a case-scenario-based questionnaire (Figure S1) to determine the influence of DNR orders on decisions about performing diagnostic tests and interventions. To maximize their validity, we developed case scenarios that described the types of acutely ill patients often encountered in hospitals in Japan. Case 1 described an 80-year-old man with an advanced stage of lung cancer who was admitted with pneumonia, Case 2 depicted an 80-year-old patient with an advanced stage of dementia who was admitted with pneumonia, and Case 3 portrayed an 80-year-old man with a non-advanced stage of heart failure who was admitted with non-life-threatening acute decompensated heart failure. Each case description was followed by questions that determined whether a physician would perform different diagnostic tests and interventions in the presence or absence of DNR orders (Table 1). CPR procedures included electrical shock treatment for VF and chest compressions for asystole. All other procedures were considered non-CPR procedures (Table 1). For Cases 1 and 2, there were 13 identical questions (Table 1). Questions 1-11 were related to non-CPR procedures, and questions 12 and 13 were related to CPR procedures. Eleven questions followed the description of Case 3, and questions 1-9 were related to non-CPR procedures, while questions 10 and 11 were related to CPR procedures (Table 1).

Because DNR orders should not affect the decision to perform non-CPR procedures, correct answers were indicated by no difference in responses to whether a physician would perform a non-CPR procedure regardless of the absence or presence of a DNR order. On the other hand, given that VF, pulseless ventricular tachycardia, asystole, and PEA indicate cardiac arrest and given that DNR orders prevent CPR, correct answers should be "no" to electrical shock for VF and "no" to chest compression for asystole in the presence of a DNR order. However, even though these answers should remain the same regardless of whether or not a physician thinks that a particular invasive procedure is futile (especially for advanced diseases such as those in Cases 1 and 2), a physician may choose not to perform this procedure regardless of the code status.

Three physicians confirmed that each question was valid and understandable and ensured that the wording within each question did not induce bias by encouraging the respondents 
Table I Questions relating to the procedures and their invasiveness for three case scenarios

\begin{tabular}{|c|c|c|c|c|c|}
\hline \multicolumn{3}{|c|}{ Cases I and 2} & \multicolumn{3}{|c|}{ Case 3} \\
\hline & Non-CPR procedure & Invasiveness & & Non-CPR procedure & Invasiveness \\
\hline QI & Computed tomography & Noninvasive & QI & Arterial blood gas & Noninvasive \\
\hline Q2 & Blood culture & Noninvasive & Q2 & Furosemide intravenously & Noninvasive \\
\hline Q3 & Antibiotic therapy & Noninvasive & Q3 & Oxygen for hypoxemia & Noninvasive \\
\hline Q4 & Normal saline & Noninvasive & Q4 & Noninvasive ventilation & Noninvasive \\
\hline Q5 & Oxygen & Noninvasive & & & \\
\hline Q6 & Norepinephrine & Moderately invasive & Q5 & Dobutamine & Moderately invasive \\
\hline Q7 & Central venous line & Moderately invasive & Q6 & Blood transfusion & Moderately invasive \\
\hline Q8 & Blood transfusion & Moderately invasive & Q7 & Intensive care unit transfer & Moderately invasive \\
\hline Q9 & Intensive care unit transfer & Moderately invasive & & & \\
\hline QI0 & Mechanical ventilation & Very invasive & Q8 & Mechanical ventilation & Very invasive \\
\hline \multirow[t]{2}{*}{ QII } & Hemodialysis & Very invasive & Q9 & Hemodialysis & Very invasive \\
\hline & CPR procedure & & & CPR procedure & \\
\hline Q12 & Electrical shock for VF & & Q10 & Electrical shock for VF & \\
\hline Q13 & Chest compression for asystole & & QII & Chest compressions for asystole & \\
\hline
\end{tabular}

Notes: Case I - advanced stage of lung cancer stage; Case 2 - advanced dementia; and Case 3 - heart failure that was not at an advanced stage. The procedures were classified into noninvasive, moderately invasive, and highly invasive procedures. See Figure SI for questionnaire.

Abbreviations: CPR, cardiopulmonary resuscitation; VF, ventricular fibrillation.

to answer a question in a particular manner. The pilot survey was tested among colleagues (physicians) and was modified according to their feedback.

\section{Survey administration}

The study protocol was approved by the institutional review boards of Tokyo Bay Urayasu Ichikawa Medical Center, Chiba, Japan, and its two affiliated community hospitals. All hospitals are acute care hospitals. Informed consent was obtained from each participant prior to participation in the study. The questionnaire was completed anonymously and was returned to the Tokyo Bay Urayasu Ichikawa Medical Center.

The effect of DNR orders on the number of non-CPR procedures that each physician was willing to perform

The number of non-CPR procedures that each physician would perform in the presence or absence of DNR orders was calculated. For Cases 1 and 2, these were calculated as percentages of the eleven questions related to non-CPR procedures, and for Case 3, these were calculated as percentages of the nine questions related to non-CPR procedures.

\section{The effect of DNR orders on the proportion of physicians who were willing to perform each non-CPR procedure}

The numbers of physicians who would perform each non$\mathrm{CPR}$ procedure in the presence or absence of DNR orders was calculated.
The effect of a procedure's invasiveness on the physician decision making modification rate

We hypothesized that DNR orders are sometimes applied as comfort measures and that they reduce physicians' willingness to perform more invasive procedures. Therefore, non-CPR procedures were categorized according to their invasiveness: noninvasive, moderately invasive, and very invasive procedures (Table 1). To maximize the validity of this classification, three independent internal medicine physicians discussed and determined the classification. We evaluated the effect of a procedure's invasiveness on the physician decision making modification rate. To estimate the physician decision making modification rate, the relative rate was calculated by dividing the number of physicians who would perform a certain procedure in the presence of a DNR order by the number of physicians who would perform a certain procedure in the absence of a DNR order. The decision making modification rate (\%) was calculated by the formula $(1-$ relative rate $) \times 100$. The higher the decision making modification rate, the more influence a DNR order had on physicians' willingness to perform a procedure.

The effect of DNR orders on the proportion of physicians who were willing to perform electric shock for VF and chest compressions for asystole

We also investigated physicians' understandings of the definition of DNR orders. As DNR orders prohibit electric shock treatment and chest compressions in the event of cardiac arrest, these CPR procedures were analyzed differently. The number of physicians who would perform electric shock 
treatment for VF and chest compressions for asystole in the presence or absence of DNR orders was determined from questions 12 and 13 for Cases 1 and 2 and from questions 10 and 11 for Case 3.

\section{Statistical analyses}

Continuous variables were expressed as medians (interquartile range, IQR), and discrete variables were summarized as percentages. Continuous variables were analyzed and compared using the Wilcoxon signed-rank test, and dichotomous variables were analyzed and compared using the $\chi^{2}$ test. Three group comparisons were performed using the one-way analysis of variance. IBM ${ }^{\circledR}$ SPSS $\AA$ software version 22 (IBM Corporation, Armonk, NY, USA) was used to perform the statistical analysis. A $P$-value $<0.05$ was considered statistically significant.

\section{Results}

\section{Participants}

About 70\% (115/161) of the questionnaires were completed and collected from the three hospitals. Of the 115 completed questionnaires, four could not be analyzed because of a lack of data; hence, 69\% (111/161) of the questionnaires were analyzed. The median (IQR) age of the respondents was $33(30-43)$ years. The median (IQR) number of postgraduate years completed by the respondents was 7 (4-16) years. Of the respondents, 84\% (93/111) were men, 56\% (62/111) were resident physicians, 44\% (49/111) were attending physicians, 39\% (44/111) were general internal medicine physicians, 10\% (12/111) were subspecialists in internal medicine, and 49\% (55/111) were specialized in areas other than internal medicine. The majority $(76 \%, 85 / 111)$ had ordered DNR orders in the past, but 53\% (59/111) were not confident about discussing code status with patients.

\section{The effect of DNR orders on the number of non-CPR procedures that each physician was willing to perform}

For Case 1, physicians were willing to perform most of the non-CPR procedures (median percent: 100\%, IQR: $90 \%-100 \%$ ) in the absence of a DNR order; however, physicians were willing to perform fewer non-CPR procedures $(72 \%, 45 \%-90 \%)$ in the presence of a DNR order $(P<0.001)$ (Figure 1). The median percent was 91\% (63\%-100\%) in the absence of a DNR order and 55\% (36\%-72\%) in the presence of a DNR order for Case $2(P<0.001)$, and $100 \%$ $(88 \%-100 \%)$ in the absence of a DNR order and $78 \%$

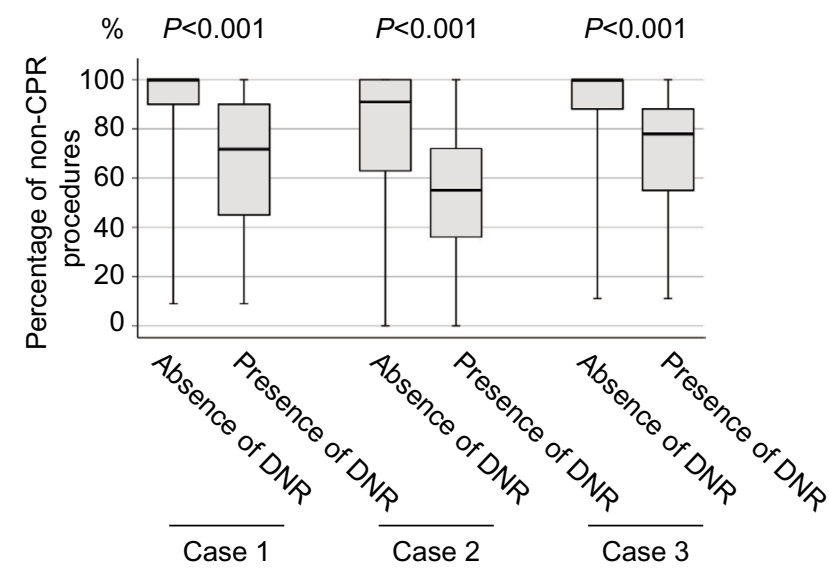

Figure I Percentages of non-CPR procedures that each physician would perform in the absence and presence of DNR orders.

Notes: The horizontal lines within the box indicate median values. The lower and upper ends of the box indicate the 25 th and 75 th percentiles, respectively. The horizontal lines below and above the box indicate the lowest and highest values, respectively. The statistical analysis was performed using Wilcoxon signed-rank test. Abbreviation: CPR, cardiopulmonary resuscitation; DNR, do not resuscitate.

$(55 \%-88 \%)$ in the presence of a DNR order for Case 3 $(P<0.001$; Figure 1).

The effect of DNR orders on the proportion of physicians willing to perform each non-CPR procedure

For Case 1, statistically significant differences were found between the number of physicians who would perform each procedure in the presence of a DNR order and the number of physicians who would perform each procedure in the absence of a DNR order (with the exception of computed tomography scans, blood cultures, and antibiotic treatments; Figure 2). Similar results were found for Case 2 (with the exception of oxygen treatment) and Case 3 (with the exception of arterial blood gas monitoring, furosemide administration, and oxygen treatment; Figure 2).

The effect of procedure invasiveness on the physician decision making modification rate

For Case 1, the average decision making modification rates associated with willingness to perform noninvasive, moderately invasive, and very invasive procedures were $5 \%, 37 \%$, and $65 \%$, respectively. In Case 2 , the rates were $10 \%, 37 \%$, and $69 \%$, respectively, while, for Case 3 , the rates were $3 \%, 31 \%$, and $63 \%$, respectively. Statistically significant differences were found in the decision making modification rates based on invasiveness $(P<0.001)$. In other words, physicians were less willing to perform procedures if they were more invasive and DNR orders were present (Figure 3). 
A Case 1

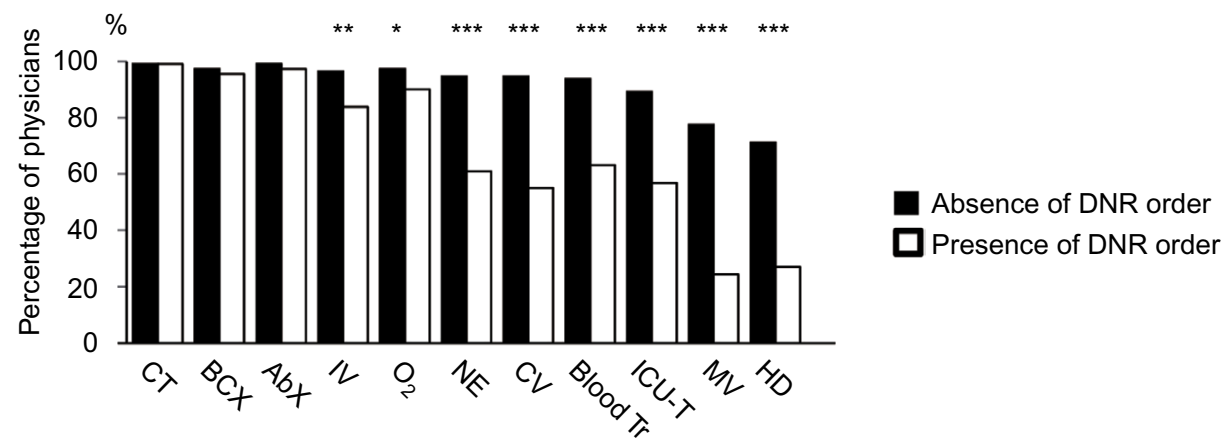

B

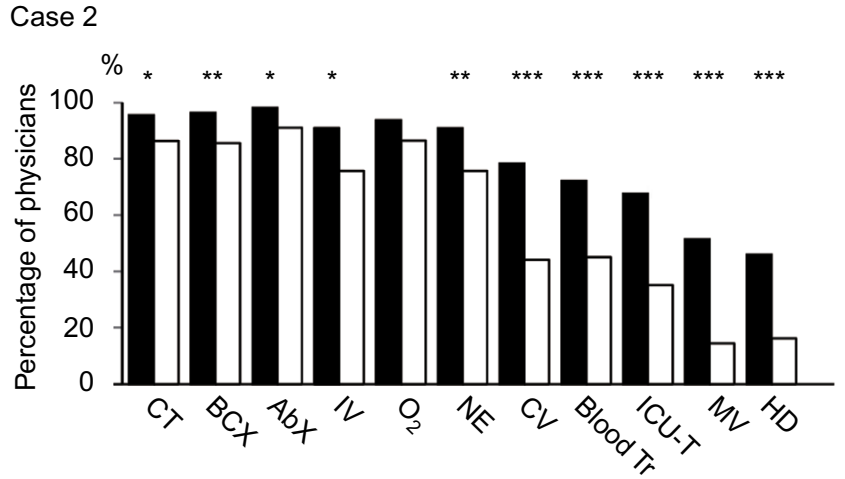

C Case 3

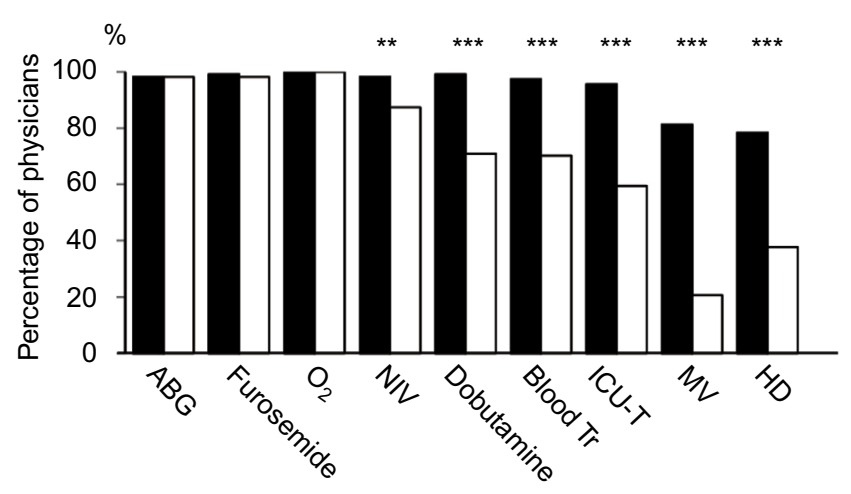

Figure 2 Percentages of physicians who would perform each procedure in the absence and presence of DNR orders.

Notes: $* P<0.05 ; * * P<0.01$; $* * * P<0.001$. (A) Case I - advanced lung cancer stage; (B) Case 2 - advanced dementia; (C) Case 3 - heart failure that was not at an advanced stage. The statistical analysis was performed using the $\chi^{2}$ test

Abbreviations: $\mathrm{AbX}$, antibiotics; IV, normal saline administration intravenously; $\mathrm{O}_{2}$, oxygen administration; NE, norepinephrine; $\mathrm{CV}$, central venous line placement; Blood Tr, blood transfusion; ICU-T, intensive care unit transfer; MV, mechanical ventilation; HD, hemodialysis; DNR, do not resuscitate; BCX, blood culture; CT, computed tomography; ABG, arterial blood gas; NIV, noninvasive ventilation.

The effect of DNR orders on the rates of physicians who were willing to perform electric shock treatment for VF and chest compressions for asystole

About one-fifth of physicians responded that they would perform electric shock treatment for VF in the presence of DNR orders (Case 1: 26\%, Case 2: 16\%, Case 3: 20\%; Figure 4). Almost all of the physicians answered that they would not perform CPR procedures for asystole in the presence of DNR orders. For Case 1, 11\% of physicians responded that they would not perform electric shock treatment for VF even in the absence of DNR orders, while $32 \%$ responded similarly in Case 2 and 12\% responded similarly in Case 3 (Figure 4).

\section{Discussion}

Although we have seen some physicians order DNR instructions for some terminally ill patients, code status orders are not mandatory for inpatient care in Japan. However, our study shows that $76 \%$ of the questionnaire respondents had ordered DNR instructions. Findings from a previous investigation showed that physicians were less likely to perform non-CPR procedures in the presence of DNR orders on patients who 


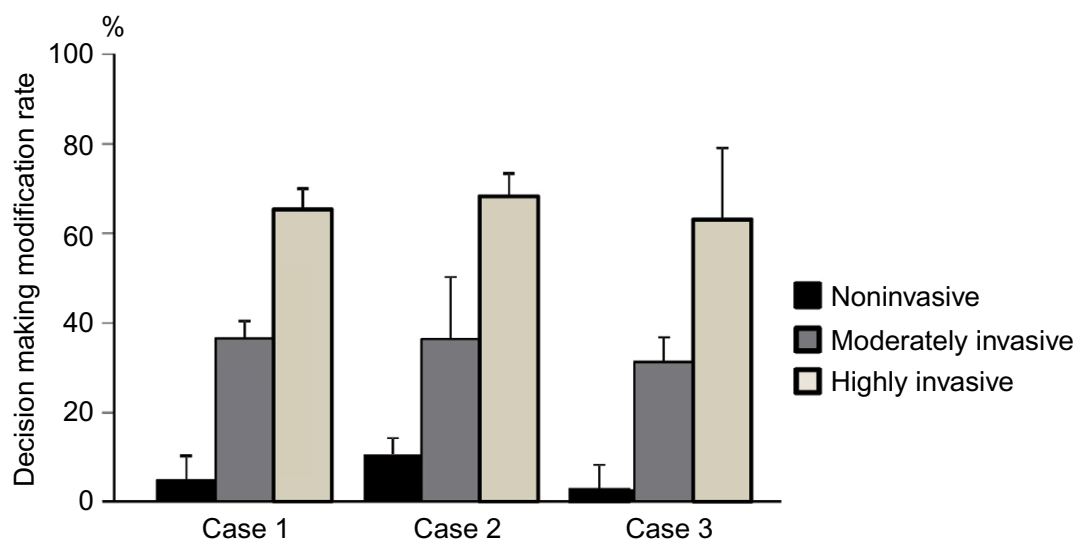

Figure 3 The effect of procedural invasiveness on physicians' decision making modification rates.

Notes: The number of physicians who would perform a certain procedure in the presence of DNR orders was divided by the number of physicians who would perform the procedure in the absence of DNR orders, and the physicians' decision making modification rate was calculated as $(I-$ this number) $\times I 00$. Error bars indicate I SD. Abbreviations: DNR, do-not-resuscitate; SD, standard deviation.
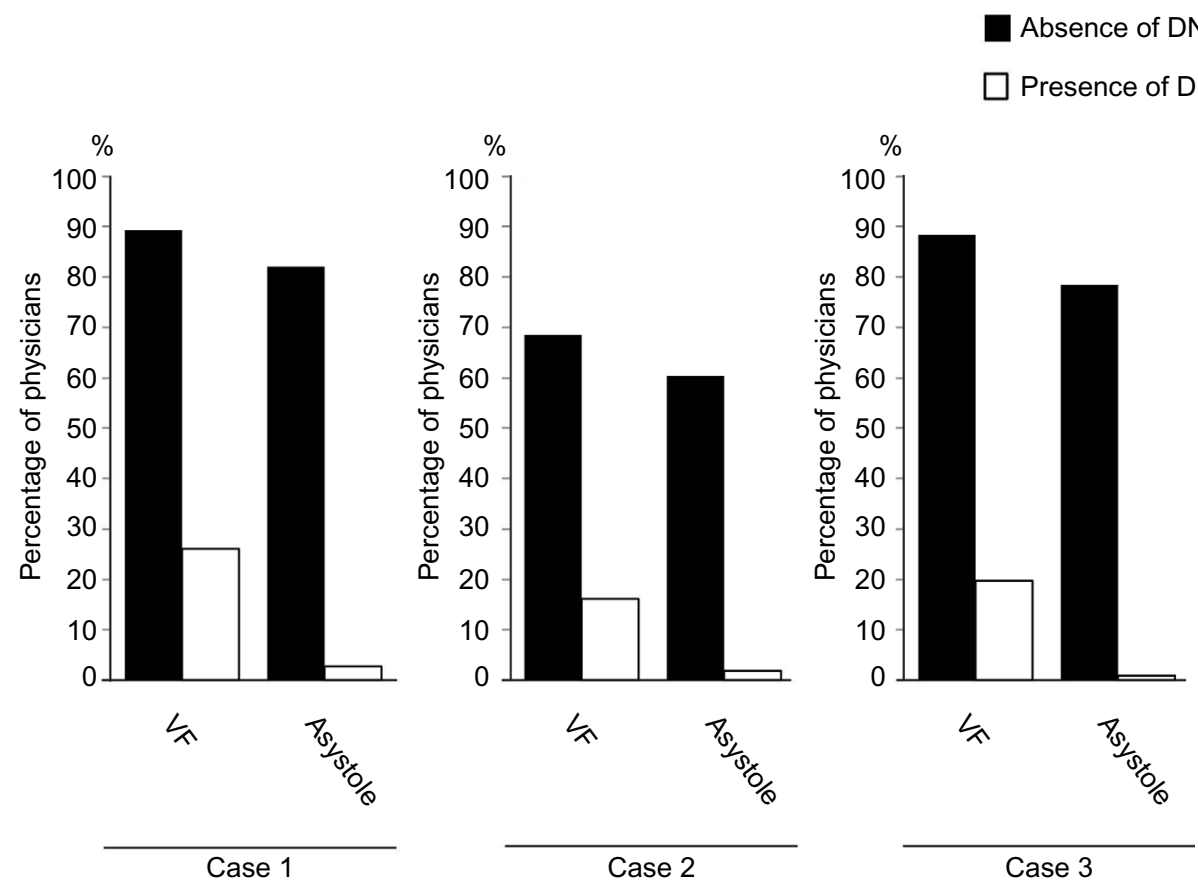

Figure 4 Percentages of physicians who would perform each CPR procedure in the absence and presence of DNR orders.

Notes: Case I - advanced lung cancer stage; Case 2 - advanced dementia; and Case 3 - heart failure that was not at an advanced stage.

Abbreviation: CPR, cardiopulmonary resuscitation; DNR, do-not-resuscitate; VF, ventricular fibrillation.

were in the final stages of cancer and HIV infection. ${ }^{3}$ We have also shown that DNR orders affect physicians' decision making processes with respect to the different non-CPR procedures and in relation to whether the patient was in the terminal stages of cancer (Case 1), was in the final stages of a noncancerous condition (Case 2), or had a noncancerous condition that was not terminal (Case 3).

Furthermore, we showed that DNR orders affected physician decision making processes to a greater extent when the non-CPR procedure was more invasive. This may have occurred because the physicians may have confused the DNR order with a comfort care order. For example, a case-scenario-based survey in the USA demonstrated that $64 \%$ of physicians equated DNR orders with comfort care/ end-of-life care rather than full care. ${ }^{9}$ It is also possible that physicians who would not perform non-CPR procedures in the presence of DNR orders might tend to have a nihilistic approach and may withdraw aggressive care too early, even though they understand that DNR orders should not be applied to non-CPR procedures. ${ }^{10}$ For example, a previous study showed that the rate at which a hospital used DNR orders for intracranial hemorrhage within 24 hours independently increased the odds of death. ${ }^{11}$ This study also showed that, after dividing the hospitals into quartiles based on 
adjusted hospital DNR order use, the hospitals with a higher rate of early DNR orders had lower rates of intubation and mechanical ventilation, craniectomy, ventriculostomy, and cerebral angiography for intracranial hemorrhage patients than hospitals with a lower rate of DNR orders. ${ }^{11}$ Hence, DNR orders may increase the risk that some physicians will not perform necessary diagnostic tests and treatments, which would be contrary to the patients' expectations. Therefore, to develop an official code status system in Japan, hospitals must clarify their policies regarding the definition of a DNR order and educate all physicians in this regard.

In all of the case scenarios, most of the doctors would not perform chest compressions for asystole in the presence of DNR orders; however, a significant number of the physicians would perform electrical cardioversion to treat $\mathrm{VF}$, even in the presence of DNR orders. It is possible that some physicians believe that VF is not cardiac arrest. Another possible explanation for this is that some physicians may think that electrical shock treatment is not invasive and is worth performing once to prolong a patient's life, even though this may be against the patient's wishes or expectations. This suggests that the definitions of cardiac arrest and DNR differ among physicians. Furthermore, witnessed VF outcomes are often good; however, the outcomes from asystole and PEA are poor, ${ }^{12,13}$ which means that, considering the results of our survey, in the presence of DNR orders, some patients with VF might survive while others might not. Therefore, whether VF is included in the definition of cardiac arrest and whether electric shock treatment should be performed for VF in the presence of a DNR order must be clarified in Japan before a code status order system is developed. Furthermore, we need to carefully articulate to patients, particularly to those who are at nonadvanced stages of diseases, exactly what DNR means and its potential consequences; otherwise DNR orders may mislead patients and physicians.

In Japan, a rapid response system (RRS) was introduced in $2008,{ }^{14}$ following the first consensus conference on medical emergency teams in the USA in $2005,{ }^{15}$ and has been expanding gradually within the country. Although there have been no official reports, at least 31 hospitals, including our hospital, have already introduced an RRS. ${ }^{14}$ Recently, it has become apparent that rapid response teams need to play a more significant role in palliative care and that the personnel sometimes need to discuss code statuses with patients. ${ }^{16}$ If the definitions of DNR and cardiac arrest differ between a patient's doctor and an RRS physician, DNR orders may harm patients; hence, DNR and cardiac arrest should be clearly defined within hospitals when an RRS is introduced.
Physicians' misunderstanding about a patient's code status preference or patients' misunderstanding of the meaning of the code status may lead to unwanted medical interventions or the withholding of desired interventions. ${ }^{17-19}$ For example, a previous study reported that patients in the ICU and their surrogates have a poor understanding of CPR, and a review of medical records revealed that, in some cases, discrepancies exist between their code status preference and the code status ordered. ${ }^{18}$ Another study demonstrated that medical students and residents do not have adequate education of end-of-life communication..$^{20}$ In our research, $53 \%$ of participants were not confident about discussing the code status with patients. Therefore, we need to educate physicians and help them develop communication skills before an official code status system is developed in Japan. Furthermore, there may also be some variation in patterns of DNR ordering between medical subspecialties. ${ }^{21}$ For example, a previous report has shown that surgeons wrote DNR orders less frequently and later in the hospital stay than internists. ${ }^{21}$ This might reflect variations in prognostication skill as well as communication skills among subspecialties. Therefore, education regarding DNR orders must be the same across subspecialties.

To the best of our knowledge, this is the first study that has demonstrated the influence of code statuses on physician decision making in Japan. However, the results of this study are limited because we only conducted the survey in three hospitals; therefore, the results cannot be extrapolated to all of the hospitals in Japan nor to other countries. On the other hand, given that there is no official guideline regarding a code status system in Japan, our study findings may alarm other hospitals, which plan to introduce code status systems. We searched the Medline and PubMed databases using the search terms "do not resuscitate" and "Bangladesh", "China", "Hong Kong”, "India”, “Indonesia”, "Iran”, "Malaysia", "Pakistan", "Philippines", "Saudi Arabia", "Singapore", "Korea”, "Taiwan", "Thailand", "Vietnam", or "Asia", and, despite some physicians using DNR orders in these countries, ${ }^{22-27}$ only one article addressed the manner in which a DNR order is interpreted. ${ }^{28}$ A Korean publication reported that DNR orders can be interpreted in two ways in Korea, namely, forbidding resuscitation and restricting care ${ }^{28}$ results which concur with our findings. Hence, other Asian countries may be alarmed by our study findings as well.

\section{Conclusion}

DNR orders affect physician decision making except in relation to CPR. The results also showed that VF is not considered to be cardiac arrest by some physicians. Therefore, before 
introducing a code status order system, precise definitions of cardiac arrest and DNR should be clarified in Japan. If not, code status orders, and especially DNR orders, may be harmful.

\section{Author contributions}

All of the authors contributed to the study's design, data analysis, drafting and revising the paper and agree to be accountable for all aspects of the work.

\section{Acknowledgment}

The authors greatly appreciate the cooperation of Dr Shigeru Katayama at Prefectural Shima Hospital and Dr Hiroaki Koike at Nikko Citizen's Hospital.

\section{Disclosure}

The authors report no conflicts of interest in this work.

\section{References}

1. Loertscher L, Reed DA, Bannon MP, Mueller PS. Cardiopulmonary resuscitation and do-not-resuscitate orders: a guide for clinicians. $\mathrm{Am}$ J Med. 2010;123:4-9.

2. Optimum care for hopelessly ill patients. A report of the Clinical Care Committee of the Massachusetts General Hospital. $N$ Engl $J$ Med. 1976;295:362-364.

3. Beach MC, Morrison RS. The effect of do-not-resuscitate orders on physician decision-making. J Am Geriatr Soc. 2002;50:2057-2061.

4. Council on Ethical and Judicial Affairs American Medical Association. Guidelines for the appropriate use of do-not-resuscitate orders. JAMA. 1991;265:1868-1871.

5. Chen JL, Sosnov J, Lessard D, Goldberg RJ. Impact of do-not-resuscitation orders on quality of care performance measures in patients hospitalized with acute heart failure. Am Heart J. 2008;156:78-84.

6. Katsetos AD, Mirarchi FL. A living will misinterpreted as a DNR order: confusion compromises patient care. J Emerg Med. 2011;40:629-632.

7. White Paper on Japanese Aging Society issued by Cabinet office, Government of Japan, 2015. Available from: http://www8.cao.go.jp/kourei/ whitepaper/index-w.html. Accessed December 31, 2015. Japanese.

8. Hazinski MF, Nolan JP, Billi JE, et al. Part 1: executive summary: 2010 international consensus on cardiopulmonary resuscitation and emergency cardiovascular care science with treatment recommendations. Circulation. 2010;122:S250-S275.

9. Ferdinando LM, Costello E, Puller J, Cooney T, Kottkamp N. TRIAD III: nationwide assessment of living wills and do not resuscitate orders. J Emerg Med. 2012;42:511-520.

10. Hemphill JC. Do-not-resuscitate orders, unintended consequences, and the ripple effect. Crit Care. 2007;11:121.

International Journal of General Medicine

Publish your work in this journal

The International Journal of General Medicine is an international, peer-reviewed open-access journal that focuses on general and internal medicine, pathogenesis, epidemiology, diagnosis, monitoring and treatment protocols. The journal is characterized by the rapid reporting of reviews, original research and clinical studies across all disease areas.
11. Hemphill JC, Newman J, Zhao S, Johnston SC. Hospital usage of early do-not-resuscitate orders and outcome after intracerebral hemorrhage. Stroke. 2004;35:1130-1134.

12. Nadkarni VM, Larkin G, Peberdy M, et al. First documented rhythm and clinical outcome from in-hospital cardiac arrest among children and adults. JAMA. 2006;295:50-57.

13. Yokoyama H, Yonemoto N, Yonezawa K, et al. Report from the Japanese registry of CPR for in-hospital cardiac arrest (J-RCPR). Circ J. 2011;75:815-822.

14. Rapid response system and in-hospital cardiac arrest registry. The Japanese Society of Intensive Care Medicine and Japanese Society for Emergency Medicine Online Resources. Available from: http:// hospital-em.net/. Accessed December 31, 2015. Japanese.

15. DeVita MA, Bellomo R, Hillman K, et al. Findings of the first consensus conference on medical emergency teams. Crit Care Med. 2006; 34:2463-2478.

16. Nelson JE, Mathews KS, Weissman DE, et al. Integration of palliative care in the context of rapid response. A report from the improving palliative care in the ICU advisory board. Chest. 2015;147:560-569.

17. Wenger NS, Phillips RS, Teno JM, et al. Physician understanding of patient resuscitation preferences: insights and clinical implications. $J$ Am Geriatr Soc. 2000;48:S44-S51.

18. Gehlbach TG, Shinkunas LA, Forman-Hoffman VL, Thomas KW, Schmidt GA, Kaldjian LC. Code status orders and goals of care in the medical ICU. Chest. 2011;139:802-809.

19. Hofmann JC, Wenger NS, Davis RB, et al. Patient preferences for communication with physicians about end-of-life decisions. Ann Intern Med. 1997;127:1-12.

20. Sullivan AM, Lakoma MD, Block SD. The status of medical education in end-of-life care: a national report. J Gen Intern Med. 2003;18:685-695.

21. Morrell ED, Brown BP, Qi R, Drabiak K, Helft PR. The do-notresuscitate order: associations with advance directives, physician specialty and documentation of discussion 15 years after the Patient Self-Determination Act. J Med Ethics. 2008;34:642-647.

22. Gouda A, Al-Jabbary A, Fong L. Compliance with DNR policy in a tertiary care center in Saudi Arabia. Intensive Care Med. 2010;36: 2149-2153.

23. Siddiqui S, Sheikh F, Kamal R. "What families want - an assessment of family expectations in the ICU". Int Arch Med. 2011;4:21.

24. Phua J, Joynt GM, Nishimura M, et al. Withholding and withdrawal of lifesustaining treatments in intensive care units in Asia. JAMA Intern Med. 2015;175:363-371.

25. Wen KY, Lin YC, Cheng JF, et al. Insights into Chinese perspectives on do-not-resuscitate (DNR) orders from an examination of DNR order form completeness for cancer patients. Support Care Cancer. 2013; 21:2593-2598.

26. Keam B, Oh DY, Lee SH, et al. Aggressiveness of cancer-care near the end-of-life in Korea. Jpn J Clin Oncol. 2008;38:381-386.

27. Sahadevan S, Pang WS. Do-not-resuscitate orders: towards a policy in Singapore. Singapore Med J. 1995;36:267-270.

28. Kim DY, Lee KE, Nam EM, et al. Do-not-resuscitate orders for terminal patients with cancer in teaching hospitals of Korea. J Palliat Med. 2007; 10:1153-1158.
The manuscript management system is completely online and includes a very quick and fair peer-review system, which is all easy to use. Visit http://www.dovepress.com/testimonials.php to read real quotes from published authors. 